Proceedings of the Edinburgh Mathematical Society (2004) 47, 365-373 (C)

DOI:10.1017/S0013091503000361 Printed in the United Kingdom

\title{
AREA INTEGRALS AND BOUNDARY BEHAVIOUR OF HARMONIC FUNCTIONS
}

\author{
MARY HANLEY \\ Department of Mathematics, University College Dublin, \\ Dublin 4, Ireland (mary.hanley@ucd.ie)
}

(Received 30 April 2003)

\begin{abstract}
This paper introduces a family of area-type integrals over cones. These are used to investigate non-tangential boundary behaviour of harmonic functions on a half-space, extending results of Stein and Brossard.
\end{abstract}

Keywords: harmonic function; boundary behaviour; area integral

2000 Mathematics subject classification: Primary 31B25

\section{Introduction}

Let $D=\left\{X=\left(X^{\prime}, x_{n}\right): X^{\prime} \in \mathbb{R}^{n-1}\right.$ and $\left.x_{n}>0\right\}$, where $n \geqslant 2$, and let $u$ be a harmonic function on $D$. For fixed $\beta>0$ and $t>0$ we define the cone

$$
\Gamma\left(Z^{\prime} ; \beta, t\right)=\left\{\left(X^{\prime}, x_{n}\right) \in \mathbb{R}^{n-1} \times(0, t):\left|X^{\prime}-Z^{\prime}\right|<\beta x_{n}\right\} \quad\left(Z^{\prime} \in \mathbb{R}^{n-1}\right),
$$

where $|\cdot|$ denotes the Euclidean norm. Also, let $\lambda_{n}$ denote the Lebesgue measure on $\mathbb{R}^{n}$. A well-known result of Stein [9] asserts that if the 'area integral'

$$
\left(\int_{\Gamma\left(Z^{\prime} ; \beta, t\right)} x_{n}^{2-n}|\nabla u(X)|^{2} \mathrm{~d} \lambda_{n}(X)\right)^{1 / 2}
$$

is finite for every $Z^{\prime}$ in a Borel set $E^{\prime} \subset \mathbb{R}^{n-1}$, then $u$ has a finite non-tangential limit $u_{\mathrm{nt}}\left(Z^{\prime}\right)$ at $\left(Z^{\prime}, 0\right)$ for $\lambda_{n-1}$-almost every point $Z^{\prime} \in E^{\prime}$. More recently, Brossard [3] used a variant of the integral in (1.1) in connection with zero boundary limits of positive harmonic functions. More precisely, he showed that if $u>0$ (and so $u_{\mathrm{nt}}\left(Z^{\prime}\right)$ exists almost everywhere), then the equivalence

$$
\int_{\Gamma\left(Z^{\prime} ; \beta, t\right)} x_{n}^{2-n}|\nabla u(X)|^{2}(u(X))^{-2} \mathrm{~d} \lambda_{n}(X)=+\infty \Longleftrightarrow u_{\mathrm{nt}}\left(Z^{\prime}\right)=0
$$

holds for almost all $Z^{\prime} \in \mathbb{R}^{n-1}$. (We say that two assertions are equivalent almost everywhere if, outside some set of measure zero, they are simultaneously either true or false.) 
The purpose of this paper is to introduce a family of area-type integrals which can be used to extend the results of Stein and Brossard and to yield some additional theorems concerning the boundary behaviour of harmonic functions.

Given a decreasing function $f:(0,+\infty) \rightarrow[0,+\infty)$ and a positive harmonic function $h$ on $D$ we define

$$
A(u, h, f)\left(Z^{\prime}\right)=\int_{\Gamma\left(Z^{\prime} ; \beta, t\right)} x_{n}^{2-n} h(X)\left|\nabla\left(\frac{u}{h}\right)(X)\right|^{2} f\left(\frac{|u(X)|}{h(X)}\right) \mathrm{d} \lambda_{n}(X) \quad\left(Z^{\prime} \in \mathbb{R}^{n-1}\right),
$$

where $f(0)$ is interpreted as $\lim _{s \rightarrow 0+} f(s)$. The expression in $(1.1)$ is $(A(u, 1,1))^{1 / 2}$ and the integral in (1.2) is $A\left(u, 1, t \mapsto t^{-2}\right)$.

Theorem 1.1. Let $u$ be a positive harmonic function on $D$ and suppose that $f$ : $(0,+\infty) \rightarrow[0,+\infty)$ is a decreasing function such that $f(s) \rightarrow+\infty$ as $s \rightarrow 0+$ and $\int_{0}^{1} s f(s) \mathrm{d} s=+\infty$. Then

$$
A(u, 1, f)\left(Z^{\prime}\right)=+\infty \Longleftrightarrow u_{\mathrm{nt}}\left(Z^{\prime}\right)=0
$$

for $\lambda_{n-1}$-almost every $Z^{\prime} \in \mathbb{R}^{n-1}$.

In addition to the case $f(s)=s^{-2}$ (Brossard's result), possible choices of $f$ in Theorem 1.1 include $s^{-2}\left[1+\log ^{+}\left(s^{-1}\right)\right]^{-a}$ when $0<a \leqslant 1$. The sharpness of the integral condition on $f$ is easily seen by considering the harmonic function $u(X)=x_{n}$. We will prove Theorem 1.1 by analytic arguments, in contrast to the probabilistic approach in $[\mathbf{3}]$.

Theorem 1.2. Let $u$ and $h$ be harmonic functions on $D$, with $h>0$, and let $f$ be the characteristic function valued 1 on $[0,1)$ and 0 on $[1,+\infty)$. Then $u$ has a finite non-tangential limit at $\left(Z^{\prime}, 0\right)$ for $\lambda_{n-1}$-almost every $Z^{\prime} \in \mathbb{R}^{n-1}$ such that $A(u, h, f)\left(Z^{\prime}\right)<+\infty$.

We note that, in the particular case where $h=1$, Theorem 1.2 relaxes the area integral hypothesis in the result of Stein mentioned earlier. This particular case can also be deduced from a result of Brossard on the density of the area integral (Theorem 2 of [4]).

Since our next result holds in a very general context, we need to introduce some notation concerning the Martin representation. (We refer to Chapter 8 of [1] for an introduction to this notion, which generalizes the well-known Poisson integral representation for a ball.) Let $\Omega$ be a connected open set with Green function $G(\cdot, \cdot)$, and let $X_{0} \in \Omega$ be our reference point. The Martin boundary (respectively, minimal Martin boundary) will be denoted by $\Delta$ (respectively, $\Delta_{1}$ ), and the Martin kernel by $M(X, Y)$. Thus

$$
M(X, Y)= \begin{cases}\frac{G(X, Y)}{G\left(X_{0}, Y\right)} & \left(X \in \Omega, Y \in \Omega \backslash\left\{X_{0}\right\}\right), \\ \lim _{Z \rightarrow Y} \frac{G(X, Z)}{G\left(X_{0}, Z\right)} & (X \in \Omega, Y \in \Delta),\end{cases}
$$

and, for each positive harmonic function $h$ on $\Omega$, there is a unique measure $\mu_{h}$ on $\Delta$ such that $\mu_{h}\left(\Delta \backslash \Delta_{1}\right)=0$ and

$$
h(X)=\int_{\Delta} M(X, Y) \mathrm{d} \mu_{h}(Y) \quad(X \in \Omega) .
$$


Theorem 1.3. Let $u$ and $h$ be harmonic functions on $\Omega$, where $h>0$, and let $X_{1} \in \Omega$. The following are equivalent:

(a) there is a decreasing function $\psi:[0,+\infty) \rightarrow(0,+\infty)$ such that $\int_{0}^{+\infty} \psi(t) \mathrm{d} t=+\infty$ and

$$
\int_{\Omega} G\left(X_{1}, X\right) h(X)\left|\nabla\left(\frac{u}{h}\right)(X)\right|^{2} \psi\left(\frac{|u(X)|}{h(X)}\right) \mathrm{d} \lambda_{n}(X)<+\infty ;
$$

(b) there is a function $g \in L^{1}\left(\mu_{h}\right)$ such that $u(X)=\int M(X, Y) g(Y) \mathrm{d} \mu_{h}(Y)$.

Corollary 1.4. Let $u$ be a harmonic function on $\Omega \backslash F$, where $F$ is a relatively closed polar subset of $\Omega$. If there is a decreasing function $\psi:[0,+\infty) \rightarrow(0,+\infty)$ such that $\int_{0}^{+\infty} \psi(t) \mathrm{d} t=+\infty$ and

$$
\int_{\Omega}|\nabla u|^{2} \psi(|u|) \mathrm{d} \lambda_{n}<+\infty
$$

then $u$ has a (unique) harmonic extension to $\Omega$.

This corollary relaxes the well-known result that polar sets are removable for harmonic functions with finite Dirichlet integral (condition (1.4) with $\psi=1$ ). Other suitable choices for $\psi(t)$ are $(1+t)^{-1}$ and $[(1+t) \log (2+t)]^{-1}$. The sharpness of the result is seen if we consider the case where $\Omega$ is the unit ball, $F=\{0\}$, and $u(X)=\log |X|$ when $n=2$ or $u(X)=|X|^{2-n}$ when $n \geqslant 3$.

In our next application of Theorem 1.3, the open set $\Omega$ is a Lipschitz domain. In this case the Martin compactification of $\Omega$ is homeomorphic to its Euclidean closure and all points of the Martin boundary are minimal (see [7], or Theorem 8.8.4 of [1] ), so we can identify $\Delta$ (and $\Delta_{1}$ ) with the Euclidean boundary $\partial \Omega$.

Corollary 1.5. Let $u$ and $h$ be harmonic functions on a Lipschitz domain $\Omega$, where $h>0$, and let $E$ be a subset of $\partial \Omega$ such that $\mu_{h}(E)=0$. If $X_{1} \in \Omega$ and there is a decreasing function $\psi:[0,+\infty) \rightarrow(0,+\infty)$ with $\int_{0}^{\infty} \psi(t) \mathrm{d} t=+\infty$ such that

$$
\int_{\Omega} G\left(X_{1}, X\right) h(X)\left|\nabla\left(\frac{u}{h}\right)(X)\right|^{2} \psi\left(\frac{|u(X)|}{h(X)}\right) \mathrm{d} \lambda_{n}(X)<+\infty,
$$

then $u$ has non-tangential limit 0 at $\sigma$-almost every point of $E$, where $\sigma$ denotes the surface area measure on $\partial \Omega$.

Our final corollary bears a superficial resemblance to Theorem 1.1. However, unlike that result, it applies to harmonic functions $u$ of variable sign.

Corollary 1.6. Let $u$ be a harmonic function on $D$ and let $\psi:[0,+\infty) \rightarrow(0,+\infty)$ be a decreasing function such that $\int_{0}^{+\infty} \psi(t) \mathrm{d} t=+\infty$. Then $u_{\mathrm{nt}}\left(Z^{\prime}\right)=0$ for $\lambda_{n-1}$-almost every $Z^{\prime} \in \mathbb{R}^{n-1}$ such that $A\left(u, x_{n}, \psi\right)\left(Z^{\prime}\right)<+\infty$.

We will prove Theorems 1.1 and 1.2 in $\S 3$, and Theorem 1.3 and its corollaries in $\S 4$, following some preparatory observations in the next section. 


\section{Preparatory material}

Theorem 2.1. Let $E^{\prime}$ be a measurable subset of $\mathbb{R}^{n-1}$ and let $u$ be a harmonic function on $D$. The following are equivalent:

(a) $u$ has a finite non-tangential limit at $\left(Z^{\prime}, 0\right)$ for $\lambda_{n-1}$-almost every $Z^{\prime} \in E^{\prime}$;

(b) $u$ is bounded in a cone of vertex $Z^{\prime}$ for $\lambda_{n-1}$-almost every $Z^{\prime} \in E^{\prime}$;

(c) $A(u, 1,1)\left(Z^{\prime}\right)<+\infty$ for $\lambda_{n-1}$-almost every $Z^{\prime} \in E^{\prime}$.

The equivalence of (a) and (b) in Theorem 2.1 was shown by Calderón [5]. We have already mentioned that Stein proved that (c) implies (a). In the same paper [9] he showed that (b) implies (c), and also proved the following lemma.

Lemma 2.2. Let $\Phi: D \rightarrow[0,+\infty)$ be a measurable function, let $E^{\prime}$ be a bounded Borel subset of $\mathbb{R}^{n-1}$ such that

$$
\int_{\Gamma\left(Z^{\prime} ; \beta, t\right)} x_{n}^{2-n} \Phi(X) \mathrm{d} \lambda_{n}(X)<+\infty \quad\left(Z^{\prime} \in E^{\prime}\right),
$$

and let $\varepsilon>0, \alpha<\beta$ and $r<t$. Then there is a compact subset $F^{\prime}$ of $E^{\prime}$ satisfying $\lambda_{n-1}\left(F^{\prime}\right)>\lambda_{n-1}\left(E^{\prime}\right)-\varepsilon$ and such that

$$
\int_{U} x_{n} \Phi(X) \mathrm{d} \lambda_{n}(X)<+\infty
$$

where $U=\bigcup_{Z^{\prime} \in F^{\prime}} \Gamma\left(Z^{\prime} ; \alpha, r\right)$.

The proof of the following elementary lemma is left to the reader.

Lemma 2.3. Let $\sum a_{m}$ be a convergent series of positive real numbers. Then there is an unbounded, strictly increasing sequence $\left(k_{m}\right)$ of positive numbers such that $\sum_{m} k_{m} a_{m}<+\infty$, and $k_{m+2}-k_{m+1} \leqslant k_{m+1}-k_{m}$ for all $m$.

\section{Proofs of Theorems 1.1 and 1.2}

\subsection{Proof of Theorem 1.1}

Suppose that $f:(0,+\infty) \rightarrow[0,+\infty)$ is a decreasing function such that $f(s) \rightarrow+\infty$ as $s \rightarrow 0+$ and $\int_{0}^{1} s f(s) \mathrm{d} s=+\infty$.

First we make the easy observation that $u_{\text {nt }}=0$ almost everywhere $\left(\lambda_{n-1}\right)$ on the set of points $Z^{\prime}$, where $A(u, 1, f)\left(Z^{\prime}\right)=+\infty$. To see this, we note that, since $u>0$, the function $u_{\mathrm{nt}}$ exists and is finite at $\lambda_{n-1}$-almost every point $Z^{\prime} \in \mathbb{R}^{n-1}$. Hence, by Theorem 2.1,

$$
A(u, 1,1)\left(Z^{\prime}\right)<+\infty \text { for } \lambda_{n-1} \text {-almost every } Z^{\prime} \in \mathbb{R}^{n-1} .
$$

The conclusion follows since, if $u_{\mathrm{nt}}\left(Z^{\prime}\right)>0$, then $f(u)$ is bounded (by $c_{Z^{\prime}}$, say) on the cone $\Gamma\left(Z^{\prime} ; \beta, t\right)$, and so $A(u, 1, f)\left(Z^{\prime}\right) \leqslant c_{Z^{\prime}} A(u, 1,1)\left(Z^{\prime}\right)$. 
Conversely, we will show that $u_{\mathrm{nt}}\left(Z^{\prime}\right)>0$ for $\lambda_{n-1}$-almost every $Z^{\prime} \in E_{k}^{\prime}$, where

$$
E_{k}^{\prime}=\left\{Z^{\prime}: A(u, 1, f)\left(Z^{\prime}\right)<+\infty \text { and }\left|Z^{\prime}\right| \leqslant k\right\} .
$$

Let $\varepsilon>0$, let $\alpha<\beta$ and $r<t$. We apply Lemma 2.2 with $\Phi=|\nabla u|^{2} f \circ u$ to see that there is a compact subset $F^{\prime}$ of $E_{k}^{\prime}$ satisfying $\lambda_{n-1}\left(F^{\prime}\right)>\lambda_{n-1}\left(E_{k}^{\prime}\right)-\varepsilon$ and such that

$$
\int_{U} x_{n}|\nabla u(X)|^{2} f(u(X)) \mathrm{d} \lambda_{n}(X)<+\infty,
$$

where $U=\bigcup_{Z^{\prime} \in F^{\prime}} \Gamma\left(Z^{\prime} ; \alpha, r\right)$.

The function $f$ is not necessarily continuous so we define $f_{1}:(0,+\infty) \rightarrow[0,+\infty)$ to be that function whose graph consists of the line segments joining the points

$$
\left\{\left(2^{-k}, f\left(2^{1-k}\right)\right): k \in \mathbb{N}\right\} \cup\{(s, 0): s \geqslant 1\} .
$$

Then $f_{1}$ is a decreasing continuous function, $f_{1} \leqslant f$ and $\int_{0}^{1} s f_{1}(s)=+\infty$. We define $\Psi:(0,+\infty) \rightarrow \mathbb{R}$ by $\Psi(x)=\int_{1}^{x} \int_{1}^{y} f_{1}(s) \mathrm{d} s \mathrm{~d} y$. Then $\Psi$ is a positive $C^{2}$ convex function that satisfies $\Psi(s) \rightarrow+\infty$ as $s \rightarrow 0+$.

Let $\chi_{U}$ denote the characteristic function of $U$ and let $a_{n}=\sigma_{n} \max \{1, n-2\}$, where $\sigma_{n}$ is the surface area of the unit sphere in $\mathbb{R}^{n}$. Using standard estimates of the Green function for $D$, it is clear from (3.1) that we can form the potential $v$ on $D$ of the measure

$$
\frac{1}{a_{n}} \chi_{U}(X)|\nabla u(X)|^{2} f_{1}(u(X)) \mathrm{d} \lambda_{n}(X) .
$$

It follows that

$$
\Delta v=-|\nabla u|^{2} f_{1}(u)=-|\nabla u|^{2} \Psi^{\prime \prime}(u)=-\triangle \Psi(u) \text { on } U .
$$

Hence $v+\Psi \circ u$ is a positive harmonic function on $U$ and so, by Théorème 10 of [2], has a finite non-tangential limit at $\left(Z^{\prime}, 0\right)$ for $\lambda_{n-1}$-almost every point $Z^{\prime} \in F^{\prime}$. It follows that $\Psi \circ u$ is non-tangentially bounded at such points. However, $\Psi(u) \rightarrow+\infty$ as $u \rightarrow 0+$. Hence $u_{\text {nt }}\left(Z^{\prime}\right)>0$ for $\lambda_{n-1}$-almost every $Z^{\prime} \in F^{\prime}$. Since $\varepsilon$ and $k$ are arbitrary, it follows that $u_{\mathrm{nt}}\left(Z^{\prime}\right)>0$ for $\lambda_{n-1}$-almost every $Z^{\prime} \in \mathbb{R}^{n-1}$, where $A(u, 1, f)\left(Z^{\prime}\right)<+\infty$.

\subsection{Proof of Theorem 1.2}

Let $u, h$ and $f$ be as in the statement of the theorem, let

$$
E_{k}^{\prime}=\left\{Z^{\prime}: A(u, h, f)\left(Z^{\prime}\right)<+\infty \text { and }\left|Z^{\prime}\right| \leqslant k\right\},
$$

and let $\alpha<\beta, r<t$ and $\varepsilon>0$. If we define $V=\{X \in D:|u(X)|<h(X)\}$, then we can apply Lemma 2.2 with $\Phi=h|\nabla(u / h)|^{2} f(|u| / h)$ to see that there is a compact subset $F^{\prime}$ of $E_{k}^{\prime}$ satisfying $\lambda_{n-1}\left(F^{\prime}\right)>\lambda_{n-1}\left(E_{k}^{\prime}\right)-\varepsilon$ and such that

$$
\int_{U \cap V} x_{n} h(X)\left|\nabla\left(\frac{u}{h}\right)(X)\right|^{2} \mathrm{~d} \lambda_{n}(X)<+\infty,
$$

where $U=\bigcup_{Z^{\prime} \in F^{\prime}} \Gamma\left(Z^{\prime} ; \alpha, r\right)$. 
Suppose that $\Psi: \mathbb{R} \rightarrow(0,+\infty)$ is a $C^{2}$ convex function such that $\Psi(t)=|t|$ on $\mathbb{R} \backslash(-1,1)$. Then $\Psi^{\prime \prime}(u / h)=0$ on $D \backslash V$. Hence, in view of $(3.2)$, we can form the potential $v$ on $D$ of the measure

$$
\frac{1}{a_{n}} \chi_{U}(X) h(X)\left|\nabla\left(\frac{u}{h}\right)(X)\right|^{2} \Psi^{\prime \prime}\left(\frac{u(X)}{h(X)}\right) \mathrm{d} \lambda_{n}(X),
$$

and

$$
\Delta v=-h\left|\nabla\left(\frac{u}{h}\right)\right|^{2} \Psi^{\prime \prime}\left(\frac{u}{h}\right)=-\Delta\left(h \Psi\left(\frac{u}{h}\right)\right) \text { on } U .
$$

The function $H=v+h \Psi(u / h)$ is positive and harmonic on $U$. Since

$$
|u|=h\left|\frac{u}{h}\right| \leqslant h \Psi\left(\frac{u}{h}\right) \leqslant v+h \Psi\left(\frac{u}{h}\right)=H \quad \text { on } U,
$$

we can apply Théorème 10 of [2] to the positive harmonic functions $H$ and $H-u$ to see that each, and thus $u$, has a finite non-tangential limit at $\left(Z^{\prime}, 0\right)$ for $\lambda_{n-1^{-a l m o s t}}$ every point $Z^{\prime}$ of $F^{\prime}$. Since $\varepsilon$ and $k$ are arbitrary, the result follows.

\section{Proof of Theorem 1.3 and corollaries}

\subsection{Proof of Theorem 1.3}

The proof of Theorem 1.3 relies, in part, on ideas from Parreau [8] (see also Theorem 9.4 .8 of $[\mathbf{1}])$.

Suppose that condition (a) of the theorem holds. Then we can form the potential $v$ on $\Omega$ of the measure

$$
\frac{1}{a_{n}} h\left|\nabla\left(\frac{u}{h}\right)\right|^{2} \psi\left(\frac{|u|}{h}\right) \mathrm{d}\left(\left.\lambda_{n}\right|_{\Omega}\right) .
$$

Define $\phi:[0,+\infty) \rightarrow[0,+\infty)$ by $\phi(x)=\int_{0}^{x} \int_{0}^{y} \psi(t) \mathrm{d} t \mathrm{~d} y$. Then $\phi$ is a convex increasing function, $x^{-1} \phi(x) \rightarrow+\infty$ as $x \rightarrow+\infty$ and $\phi^{\prime \prime}=\psi$, and we have

$$
\Delta v=-h\left|\nabla\left(\frac{u}{h}\right)\right|^{2} \psi\left(\frac{|u|}{h}\right)=-\Delta\left(h \phi\left(\frac{|u|}{h}\right)\right) \quad \text { on } \Omega .
$$

Let $w=v+h \phi(|u| / h)$. Then $w$ is a positive harmonic function on $\Omega$ which majorizes $h \phi(|u| / h)$ and thus $|u| \leqslant h \phi^{-1}(w / h)$. But $h \phi^{-1}(w / h)$ is a superharmonic function on $\Omega$ (see Theorem 3.4.3 of [1] ), so it follows that the subharmonic function $|u|$ has a harmonic majorant $H$ such that $H \leqslant h \phi^{-1}(w / h)$. Whence $h \phi(H / h) \leqslant w$. Let $m \in \mathbb{N}$ and define $b_{m}=\sup \{t / \phi(t): t \geqslant m\}$. Then

$$
t \leqslant b_{m} \phi(t)+m \quad(t>0, m \in \mathbb{N})
$$

and

$$
H \leqslant b_{m} h \phi\left(\frac{H}{h}\right)+m h \leqslant b_{m} w+m h .
$$


Let $\nu_{H}$ denote the singular part of $\mu_{H}$ with respect to $\mu_{h}$. We can choose sets $A$ and $B$ such that $\nu_{H}(B)=0=\mu_{h}(A)$ and $A \cup B=\Delta_{1}$, and then observe that

$$
\nu_{H}\left(\Delta_{1}\right)=\nu_{H}(A)=\mu_{H}(A) \leqslant b_{m} \mu_{w}(A) \text { for all } m .
$$

Since $b_{m} \rightarrow 0$ as $m \rightarrow+\infty$, we have $\nu_{H}\left(\Delta_{1}\right)=0$. Similarly, $\nu_{H-u}\left(\Delta_{1}\right)=0$, since, from $(4.1)$,

$$
0 \leqslant H-u \leqslant 2 H \leqslant 2 b_{m} w+2 m h .
$$

If we define $\mu_{u}=\mu_{H}-\mu_{(H-u)}$, then $\mu_{u}$ is absolutely continuous with respect to $\mu_{h}$ and so, by the Radon-Nikodým Theorem, condition (b) holds.

Conversely, suppose that (b) holds. Our first step is to show that there is an increasing convex $C^{2}$ function $\phi:[0,+\infty) \rightarrow[0,+\infty)$ such that $\phi^{\prime \prime}$ is decreasing, $t^{-1} \phi(t) \rightarrow+\infty$ as $t \rightarrow+\infty$ and $\phi(|g|)$ is $\mu_{h}$-integrable. (If $g$ is bounded, then $\phi(t)=t^{2}$ will suffice.) Let

$$
A_{m}=\{Y \in \Delta: m-1 \leqslant|g(Y)|<m\}, \quad m \in \mathbb{N} .
$$

Then $\sum_{m} m \mu_{h}\left(A_{m}\right)<+\infty$ and it follows from Lemma 2.3 that there is an unbounded strictly increasing sequence $\left(k_{m}\right)$ of positive numbers such that

$$
k_{m+2}-k_{m+1} \leqslant k_{m+1}-k_{m} \leqslant k_{1} \text { for all } m
$$

and $\sum_{m} m k_{m} \mu_{h}\left(A_{m}\right)<+\infty$. We define $\psi:[0,+\infty) \rightarrow(0,+\infty)$ to be that function whose graph comprises the line segments joining the points $\left\{\left(0, k_{1}\right)\right\} \cup\left\{\left(m, k_{m+1}-k_{m}\right)\right.$ : $m \in \mathbb{N}\}$. Then $\psi$ is a decreasing function and

$$
\int_{0}^{s} \psi(t) \mathrm{d} t \geqslant k_{[s]}-k_{1} \rightarrow+\infty \quad \text { as } s \rightarrow+\infty
$$

Now we define $\phi:[0,+\infty) \rightarrow[0,+\infty)$ by $\phi(t)=\int_{0}^{t} \int_{0}^{y} \psi(s) \mathrm{d} s \mathrm{~d} y$ and observe that

$$
0 \leqslant \phi(t) \leqslant \sum_{i=1}^{m} k_{i} \quad(t \leqslant m, m \geqslant 1)
$$

Clearly, $\phi$ is an increasing convex function such that $\phi^{\prime \prime}=\psi$, and $t^{-1} \phi(t) \rightarrow+\infty$ as $t \rightarrow+\infty$. Further, $\phi(|g|)$ is $\mu_{h}$-integrable since

$$
\int_{A} \phi(|g|) \mathrm{d} \mu_{h} \leqslant \sum_{m} \phi(m) \mu_{h}\left(A_{m}\right) \leqslant \sum_{m} m k_{m} \mu_{h}\left(A_{m}\right)<+\infty .
$$

Given $X \in \Omega$, we apply Jensen's inequality with the unit measure $(M(X, \cdot) / h(X)) \mathrm{d} \mu_{h}$ to obtain

$$
\begin{aligned}
\phi\left(\frac{|u(X)|}{h(X)}\right) & \leqslant \phi\left(\frac{\int M(X, Y)|g(Y)| \mathrm{d} \mu_{h}(Y)}{h(X)}\right) \\
& \leqslant \int \frac{M(X, Y)}{h(X)} \phi(|g(Y)|) \mathrm{d} \mu_{h}(Y) .
\end{aligned}
$$


Hence, the subharmonic function $h \phi(|u| / h)$ has a harmonic majorant $H_{1}$ given by

$$
H_{1}=\int M(\cdot, Y) \phi(|g(Y)|) \mathrm{d} \mu_{h}(Y) .
$$

The non-negative superharmonic function $H_{1}-h \phi(|u| / h)$ has Riesz decomposition $v+H_{2}$, where $v$ is a potential on $\Omega$ and $H_{2}$ is harmonic there. Hence

$$
\Delta v=-\Delta\left(h \phi\left(\frac{|u|}{h}\right)\right)=-h\left|\nabla\left(\frac{u}{h}\right)\right|^{2} \phi^{\prime \prime}\left(\frac{|u|}{h}\right)=-h\left|\nabla\left(\frac{u}{h}\right)\right|^{2} \psi\left(\frac{|u|}{h}\right)
$$

and (a) follows. This completes the proof of Theorem 1.3.

\subsection{Proof of Corollary 1.4}

Let $u$ be a harmonic function on $\Omega \backslash F$, where $F$ is a relatively closed polar subset of $\Omega$. We choose the reference point $X_{0}$ for the Martin compactification of $\Omega$ to belong to $\Omega \backslash F$. Then, for each $Y \in \Delta_{1} \cup F$, the Martin kernel of $\Omega \backslash F$ is equal to the Martin kernel $M(X, Y)$ of $\Omega$ restricted to $\Omega \backslash F$, and the minimal Martin boundary of $\Omega \backslash F$ is $\Delta_{1} \cup F$ (see Theorem 9.5.1 (i) of [1]). Now, suppose that there is a function $\psi$ as described in Corollary 1.4. It follows that condition (a) of Theorem 1.3, with $h=1$, holds. So, by condition (b) of Theorem 1.3, there is a $\mu_{1}$-integrable function $g$ on $\Delta_{1} \cup F$ such that

$$
u(X)=\int_{\Delta_{1} \cup F} M(X, Y) g(Y) \mathrm{d} \mu_{1}(Y) \quad(X \in \Omega \backslash F) .
$$

Since $\mu_{1}(F)=0$ the function $u$ has a harmonic extension to $\Omega$ which is clearly unique.

\subsection{Proof of Corollary 1.5}

Now let $u, h, E$ and $\psi$ be as in Corollary 1.5, and suppose that (1.5) holds. Then condition (a), and hence (b), of Theorem 1.3 holds. Since we can identify both $\Delta$ and $\Delta_{1}$ with the Euclidean boundary $\partial \Omega$, there is a $\mu_{h}$-integrable function $g$ on $\partial \Omega$ such that

$$
u(X)=\int_{\partial \Omega} M(X, Y) g(Y) \mathrm{d} \mu_{h}(Y) \quad(X \in \Omega) .
$$

It is sufficient to consider the case where $u \geqslant 0$, since $u$ is the difference of the two non-negative harmonic functions

$$
\int_{\partial \Omega} M(\cdot, Y) g^{+}(Y) \mathrm{d} \mu_{h}(Y) \text { and } \int_{\partial \Omega} M(\cdot, Y) g^{-}(Y) \mathrm{d} \mu_{h}(Y) .
$$

Now $\mu_{u}(E)=\int_{E} g \mathrm{~d} \mu_{h}=0$, so $u$ has minimal fine limit 0 at $\mu_{1}$-almost every point of $E$ (see Corollary 9.4.2 of [1]). Since $\Omega$ is Lipschitz, Theorem 5.5 of [7] asserts that $u$ has non-tangential limit 0 at every point of $E$ where it has a minimal fine limit 0 . Further, by Theorem 3 of $[\mathbf{6}]$, the measures $\mu_{1}$ and $\sigma$ on $\partial \Omega$ are mutually absolutely continuous. The result follows. 


\subsection{Proof of Corollary 1.6}

It remains to establish Corollary 1.6. Let $u$ and $\psi$ be as in the statement of the result, let

$$
E_{k}^{\prime}=\left\{Z^{\prime}: A\left(u, x_{n}, \psi\right)\left(Z^{\prime}\right)<+\infty \text { and }\left|Z^{\prime}\right| \leqslant k\right\}
$$

and let $\alpha<\beta, r<t$ and $\varepsilon>0$. We can apply Lemma 2.2 with

$$
\Phi(X)=x_{n}\left|\nabla\left(\frac{u(X)}{x_{n}}\right)\right|^{2} \psi\left(\frac{u(X)}{x_{n}}\right)
$$

to see that there is a compact subset $F^{\prime}$ of $E_{k}^{\prime}$ satisfying $\lambda_{n-1}\left(F^{\prime}\right)>\lambda_{n-1}\left(E_{k}^{\prime}\right)-\varepsilon$ and such that

$$
\int_{U} x_{n}^{2}\left|\nabla\left(\frac{u(X)}{x_{n}}\right)\right|^{2} \psi\left(\frac{u(X)}{x_{n}}\right) \mathrm{d} \lambda_{n}(X)<+\infty,
$$

where $U=\bigcup_{Z^{\prime} \in F^{\prime}} \Gamma\left(Z^{\prime} ; \alpha, r\right)$. The number of components in $U$ is finite since $F^{\prime}$ is compact, so, without loss of generality, we can assume that $U$ is connected. It is not difficult to check that $U$ is a Lipschitz domain. Taking standard estimates for the Green function on $D$ (and noting that the Green function for $D$ at $(X, Y) \in U \times U$ majorizes the Green function for $U$ at $(X, Y)$ ), it is clear from (4.2) that condition (1.5) of Corollary 1.5 holds with $h(X)=x_{n}$ and $\Omega=U$. Since

$$
\mu_{x_{n}}\left(F^{\prime} \times\{0\}\right)=\int_{F^{\prime} \times\{0\}} x_{n} \mathrm{~d} \mu_{1}(X)=0,
$$

it follows from Corollary 1.5 that $u_{\mathrm{nt}}\left(Z^{\prime}\right)=0$ for $\lambda_{n-1}$-almost every $Z^{\prime} \in F^{\prime}$. Since $\varepsilon$ and $k$ are arbitrary, the result follows.

Acknowledgements. It is a pleasure to thank Professor Stephen Gardiner for many helpful suggestions during the writing of this paper.

\section{References}

1. D. H. Armitage and S. J. Gardiner, Classical potential theory (Springer, 2001).

2. M. Brelot and J. L. Doob, Limites angulaires et limites fines, Annls Inst. Fourier $\mathbf{1 3}$ (1963), 395-415.

3. J. Brossard, Intégrale d'aire et support d'une mesure positive, C. R. Acad. Sci. Paris Sér. I 296 (1983), 231-232.

4. J. Brossard, Densité de l'intégrale d'aire dans $\mathbb{R}_{+}^{n+1}$ et limites non tangentielles, Invent. Math. 93 (1988), 297-308.

5. A. P. CALDERón, On the behaviour of harmonic functions at the boundary, Trans. Am. Math. Soc. 68 (1950), 47-54.

6. B. E. J. DahlBerg, Estimates of harmonic measure, Arch. Ration. Mech. Analysis 65 (1977), 275-288.

7. R. A. Hunt and R. L. Wheeden, Positive harmonic functions on Lipschitz domains, Trans. Am. Math. Soc. 147 (1970), 507-527.

8. M. PARREAU, Sur les moyennes des fonctions harmoniques et analytiques et la classifcation des surfaces de Riemann, Annls Inst. Fourier 3 (1951), 103-197.

9. E. M. Stein, On the theory of harmonic functions of several variables, II, Acta Math. 106 (1961), 137-174. 